\title{
A Class-Oriented Strategy for Features Extraction from Multidate ASTER Imagery
}

\section{Nicola Crocetto ${ }^{1}$ and Eufemia Tarantino ${ }^{2, *}$}

1 Department of Civil Engineering, Second University of Naples, via Roma n. 29, Aversa (CE), 81031, Italy; E-Mail: nicola.crocetto@unina2.it

2 Department of Highway and Transportation, Section Geomatics, Polytechnic University of Bari, via Orabona n.4, Bari, 70125, Italy

* Author to whom correspondence should be addressed; E-Mail: e.tarantino@poliba.it; Tel. / Fax: +39-0805963417.

Received: 23 October 2009; in revised form: 20 November 2009 / Accepted: 26 November 2009 / Published: 30 November 2009

\begin{abstract}
In this paper we propose a hybrid classification method, adopting the best features extraction strategy for each land cover class on multidate ASTER data. To enable an effective comparison among images, Multivariate Alteration Detection (MAD) transformation was applied in the pre-processing phase, because of its high level of automation and reliability in the enhancement of change information among different images. Consequently, different features identification procedures, both spectral and object-based, were implemented to overcome problems of misclassification among classes with similar spectral response. Lastly, a post-classification comparison was performed on multidate ASTER-derived land cover (LC) maps to evaluate the effects of change in the study area.
\end{abstract}

Keywords: class-oriented classification; MAD calibration; TIR ASTER bands; change detection

\section{Introduction}

Over the last decades remote sensing has improved our ability to interpret environmental processes acting on the terrestrial globe. However, those interested in acquiring information on land cover using remotely sensed data must consider a number of issues, not least the identification of the most appropriate dataset(s) for the task in hand. 
Recent diffusion of Very High spatial Resolution satellite VHR sensors (i.e., IKONOS, QUICKBIRD, etc.) has widened visual and analytical interpretation of any territory at large scale, but the low spectral capability of their four VIR channels in some cases limit their effective use. ASTER satellite system has wide spectral coverage and relatively high spatial resolution and it allows to distinguish a variety of surface materials, reducing problems in the interpretation of some lower resolution data resulting from mixed pixels The Advanced Spaceborne Thermal Emission and Reflection Radiometer (ASTER) is a multispectral sensor system that was launched on board NASA's Terra spacecraft in December 1999. ASTER covers a wide spectral region with 14 bands ranging from visible to thermal infrared bands with high spatial, spectral and radiometric resolution. An additional backward-looking near-infrared band provides stereo coverage [1,2].

In recent years pixel-based or object-based classifications on single acquisition ASTER data have been investigated for land cover studies [3,4,5], but currently some unresolved questions on their use still persist, i.e., misclassifications among classes with similar spectral reflectance in arid zones (built-up area, outcrop, quarries, etc.). In these cases class-oriented hybrid approaches can improve the quality of results, by providing a combination of various image processing methods [6]. Multiple data collections are yet necessary to monitor environmental processes, and the choice of a reliable change detection technique on radiometrically homogeneous data can help identify land cover transformations [7].

The purpose of this work was to develop a class-oriented classification approach that effectively integrates multichannel spectral information into the classification process, combining pixel-based and object-based strategies to prevent misclassifications among classes with similar spectral response on multiple ASTER data. To such purpose, thermal information and/or the Soil Adjusted Vegetation Index (SAVI) were taken into account in the segmentation phase. VNIR and SWIR data were normalized with the Multivariate Alteration Detection (MAD) technique and co-georeferenced to achieve homogenization and record multiple date imagery in an unique digital information environment. TIR data were separated into temperature and emissivity values using the emissivity normalization technique [8], in order to derive the spatial distribution (pattern) of thermal objects in the analyzed territory. Lastly, a post-classification comparison was performed on multiple date ASTER-derived land cover (LC) maps.

The paper is organized in sections as follows: Section 2 reviews pixel-based and object-based approaches for change detection analysis; Section 3 examines radiometric normalization methods; Section 4 describes data collection and pre-processing performed for this study; Section 5 illustrates the class-oriented classification strategy proposed; Sections 6 and 7 conclude the paper by discussing on the results.

\section{Pixel-Based and Object-Based Approaches for Change Detection}

Many feature extraction techniques are available, but the choice of the best method depends on the context of the application and the satellite data chosen. Commonly, techniques that include a range of statistical, structural and neural approaches are used based on per-pixel classifications. When standard procedures of per-pixel multispectral classification are applied to medium spatial resolution, as in the case of ASTER data, these imagery sources are likely to generate problems with relation to the degree of heterogeneity of the materials in the scene. In order to solve these problems, some 
post-classification procedures were investigated on lower spatial resolution data on the basis of intrinsic contextual information [9]. Although a reduction of noise in the classified images was obtained, substantial improvements in the overall accuracy of results were not observed. Moreover, a loss of meaningful information in classified data was shown as a result of geometric and dimensional noncorrespondence of real elements with moving window implementation matrixes (e.g., with majority logical filters). Such methods require extensive editing to be performed on classified images in order to store them in GIS databases [10].

With the advent of VHR (Very High Resolution) sensors many techniques [11,12] were studied. One of the first methods was per-field classification (so called because fields, as opposed to pixels, are classified as independent units), which takes into account spectral and spatial properties of the imagery, size and shape of the fields and the land cover classes chosen. In fact, this approach requires a priori information on the boundaries of the objects within the image, and therefore positively contributes to the classification of remote sensing imagery of high to medium geometric resolution. Problems arise in cases where no boundaries are readily available or when those boundaries should be exact [13].

Image segmentation can offer a solution. In many cases, image analysis leads to the identification of meaningful objects only when the image is segmented in 'homogenous' areas [14-16] which is commonly known as object-based image analysis (OBIA). Rich spectral and spatial information inherent in satellite data is jointly utilized to enhance the chance of correctly labeling pixels in the decision making process [17]. The most evident differences between pixel-based image analysis and object-based image analysis are: (1) the basic processing units are image objects, or segments, not single pixels; (2) classifiers in object-oriented image analysis are soft classifiers based on fuzzy logic, which has the apparent advantage of expressing any uncertainties in the description of classes [18]. The resulting segmentation generates homogeneous objects and should be reproducible and universal, thus allowing application to a large variety of data [19]. In recent years, successful application of OBIA concepts, methods and tools has been reported in different application domains. This is connected to the rising of GEOBIA as a GIScience discipline devoted to developing automated methods to partition remote sensing images into meaningful image-objects, and assessing their contextual and spectral characteristics at different spatial and temporal scales [20,21].

Change detection is the process of identifying differences in the state of an object or phenomenon by observing it at different times [22]. It encompasses a broad range of methods used to identify, describe and quantify differences between images of the same scene at different times or under different conditions. Macleod and Congalton [23] list four aspects of change detection which are important when monitoring natural resources: detecting that changes have occurred, identifying the nature of the change, measuring the areal extent of the change and assessing the spatial pattern of the change. Coppin and Bauer [24] summarize eleven different change detection algorithms that were found to be documented in the literature. These include: (1) Monotemporal change delineation; (2) Post-classification comparison; (3) Multidimensional temporal feature space analysis; (4) Composite analysis; (5) Image differencing; (6) Image rationing; (7) Multitemporal linear data transformation; (8) Change vector analysis; (9) Image regression; (10) Multitemporal biomass index; (11) Background subtraction. 
This research is based on the use of post-classification which is the most common and intuitive change detection method. Post-classification comparison determines the difference between independently classified images from each of the dates concerned. It is the only method in which "from" and "to" classes can be calculated for each changed pixel. The change image can be a simple "mask" image, where a new value is given if there is a change, and zero is returned if the pixel values are the same (unchanged). The algorithm to produce the difference image can also be programmed to be selective in what change is shown or indicate the original class [25].

\section{Radiometric Normalization Methods}

All the above methods, when used in multidate analysis, do not consider the issue of data homogenization in change detection. The final comparison is usually made among classification results based on raw satellite data. Artifacts in the uncorrected data could heavily alter the final results because it does not consider different local atmospheric conditions and sensor characteristics. Radiometric normalization is important in land cover classifications and for many other applications, such as image mosaicing [26], tracking vegetation indices over time [27] etc.

Previous literature shows that several statistical techniques can be applied for the identification of changes on a pixel basis, e.g., calculating spectral or texture pixel values, estimating changes in transformed pixel values or identifying changes in pixel class memberships. The limits of these techniques are connected to the temporal stability of sensor calibration, to the level of correlation of spectral bands, and to the geometry of sun-earth-sensors. If these elements vary between image data, an effective comparison among images is not possible, because the data analyzed do not have a common radiometric reference.

Absolute radiometric correction of multitemporal satellite imagery requires atmospheric corrections. When atmospheric correction parameters for imagery are not available or absolute surface radiances are not necessary, an alternative can be offered by relative calibration (by many authors referred to as "normalization") of satellite images to a master scene, based on radiometric information intrinsic to the same images [28].

To such purpose, Jensen [29] suggested the utilization of Multiple date Image Normalization Using Regression method. This method involves the selection of ground targets whose reflectance values are considered as constant over time, otherwise named as "Pseudo-Invariant Features" (PIFs) by Schott et al. [30]. With radiometric normalization, the selection of such ground targets is entirely dependent on the abilities and knowledge of the analyst, and on an adequate degree of spatial and spectral resolution of the data. Besides the loss of accuracy in manual identification of invariant targets, a further limit is the case in which satellite data are affected by intrinsic radiometric problems due to different climatic conditions experienced during the acquisition phase, as cloud or snow covers.

In order to remedy to such inconveniences by identifying potential no-change pixels a priori, Schott et al. [30] proposed the utilization of band ratios and Hall et al. [31] of Tasseled cap transformation. Du et al. [32] applied PCA (Principal Components Analysis) to assist the user in the selection of invariant features or no-change pixels.

Although the principle is similar (invariant pixels are used in the regression approach), MAD transformation [33,34] is fully automatic. Moreover, this procedure is invariant compared with linear 
effects caused by atmospheric conditions and sensor calibration [35].The main advantage of MAD is the automatic identification of "no change pixels". Furthermore, basic data come from the same image, without any interference of unfavorable climatic conditions or any type of noise/variation in terms of reflectance.

The MAD procedure is based on a classical statistical transformation referred to as "correlation analysis", which enhances change information in the difference, as described below. Invariant pixels are selected by means of the simple image difference $\boldsymbol{D}$ (with $\boldsymbol{F}$ and $\boldsymbol{G}$ matrixes or random vectors) acquired on two different dates $\left(\mathrm{t}_{1}, \mathrm{t}_{2}\right)$ :

$$
\boldsymbol{D}=\boldsymbol{F}-\boldsymbol{G}=\left[F_{1}-G_{1} \ldots F_{k}-G_{k}\right]^{T}=a^{\mathrm{T}} F-b^{\mathrm{T}} G
$$

with:

where $k=$ band number.

$$
\boldsymbol{F}=\left[F_{1} \ldots F_{k}\right]^{T} \text { and } \mathbf{G}=\left[G_{1} \ldots G_{k}\right]^{T}
$$

Analogously to principal component transformation, the vectors $a$ and $b$ are sought subject to the condition that the variance of $\boldsymbol{D}$ is maximized and subject to the constraints that:

$$
\operatorname{var}\left(a^{T} F\right)=\operatorname{var}\left(b^{T} G\right)=1
$$

Consequently, the difference image D contains the maximum spread in its pixel intensities and-provided that this spread is due to real changes between $t_{1}$ and $t_{2}$-therefore $\boldsymbol{D}$ corresponds to maximum change information. Determining the vectors $a$ and $b$ as above is a standard statistical procedure, equivalent to the so-called generalized eigenvalue problem. For a given number of bands $N$, the procedure returns $N$ eigenvalues, $N$ pairs of eigenvectors and $N$ orthogonal (uncorrelated) difference images, referred to as "MAD variants" [36].

Since relevant changes of man-made structures will generally not be evident as seasonal vegetation changes, or statistical image noise, they expectedly concentrate in the higher order components (in order of increasing variance). The calculations involved are invariant under affine transformation of the original image data. Assuming that changes in the overall atmospheric conditions or in sensor calibrations are approximately equivalent to affine transformations of pixel intensities, the method is insensitive to both of these effects [35].

\section{Data Collection and Pre-Processing}

\subsection{Geometric Correction}

Two summer season ASTER Level 1A data (acquisition Dates of 2003-06-24 and 2007-07-14; Time 9:45) with similar atmospheric conditions of a flat coastal territory of Apulia (Italy) were analyzed to validate the proposed method. The Italian name for the whole area is 'Murge' which includes three subregions parallel to the coast: Coastal Murgia, Lower Murgia, and Upper Murgia. The intensity of agricultural land use and the population density generally decrease in this area as we move from the coast inland. The study area is located in the coastal subregion, a narrow zone, alternating in width from some hundred metres to a few kilometres. This area is rather intensively cultivated due to the comparatively deep soils and easy accessibility of the groundwater. Crops are grown on a 
rotational basis with a wide selection of vegetables and fruits. A large portion of the irrigated land features vineyards and olive groves as the most widespread crops.

To facilitate the comparison of multidata imageries it was necessary to register both data sets in a single map coordinate system, in this case Universal Transverse Mercator projection (zone 33) and datum WGS84. A high (3rd) order polynomial transformation for rectification was executed to consider the relief of certain subareas. In this process the identification of 20 common Ground Control Points (GCP), regularly distributed in the study area and obtained by recognising permanent and detailed elements at imagery scale, was planned. A planimetric accuracy of 0.896 pixels (RMS value lower than 1 pixel) was achieved for the three visible-near infrared (VNIR) bands. This residual was reduced to 0.448 pixels for the six shortwave infrared (SWIR) bands, even though all 20 GCPs were retained in the transformation.

Figure 1. Location of the study area and original subsets of ASTER data: acquisition dates of 2003-06-24 (left) and 2007-07-14 (right).

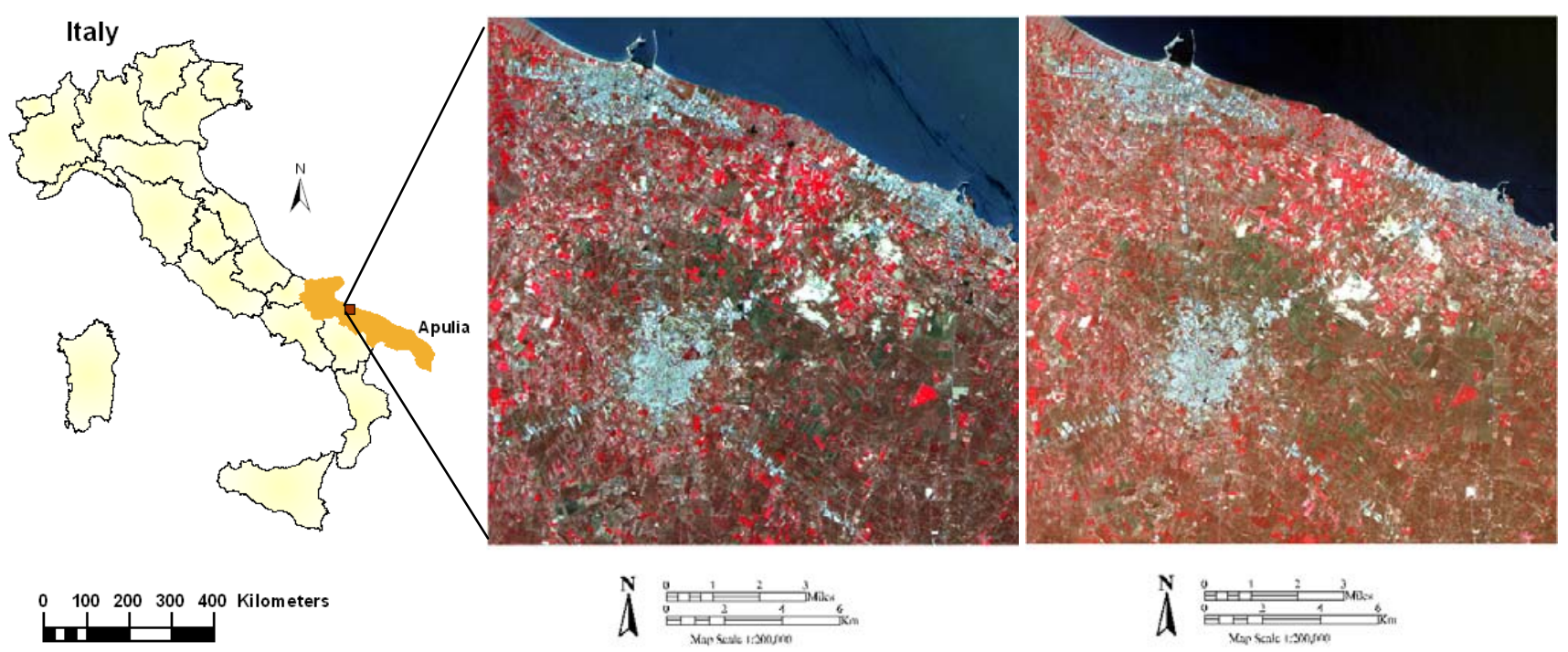

All the geo-referenced bands were output at $15 \mathrm{~m}$ using the Nearest Neighbour resampling method during image transformation, in order to not alter the radiometric content of images significantly. The VNIR bands were then stacked with the resampled SWIR bands to form a composite image. To enable a better identification of some classes, TIR data were distinguished into one brightness temperature (Figure 3) and five emissivity images using the emissivity-temperature separation technique described by Realmuto (1990) [8], The Land Surface Temperature (LST) was then geo-referenced and resampled with an output of $15 \mathrm{~m}$ for its further integration in the classification phase.

Subsequently, a subimage of 1,200 rows by 1,200 columns was delimited from the full-scene image, which corresponded to a ground area of $18 \times 18 \mathrm{~km}^{2}$, encompassing a diversified morphology in which the rural, quarry and urban land uses dominate the territories of three medium size towns (Andria, Corato, Trani) of the Apulian region (Figure 1). 


\subsection{Radiometric Normalization of VNIR and SWIR Data}

The next step in the pre-processing phase consisted in radiometric calibration aimed at the homogenization of multidate VNIR and SWIR data.

First, the single VNIR/SWIR datasets of the "master" image (2003) and the data to be normalized or "target" image (2007) were atmospherically corrected by means of ENVI Fast Line-of-sight Atmospheric Analysis of Spectral Hypercubes (FLAASH) module which includes the MODTRAN-4 [37]. Hence both datasets were compensated for atmospheric effects retrieving spectral reflectance from multispectral radiance images. All historical climatic data were acquired from Bari Palese meteorological station located $50 \mathrm{Km}$ from the study area.

As a second step, the MAD technique was implemented on reflectance images by using Change Detection and automatic radiometric calibration (CDSAT) based on the iteratively reweighted MAD (IR-MAD) transformation-ENVI plug-in. Such procedure enabled the identification of invariant pixels, while the calibration parameters were determined with orthogonal regression. Due to its completely automatic operation, and as parameters are free and fast, the MAD based normalization technique was favoured in comparison with the definition of decision thresholds or the individuation of PIF (Pseudo Invariant Features) with subjective criterions using similar manual techniques. For, with MAD transformation basic data totally come from the same image, without interference of unfavorable climatic conditions or every type of noise/variation in terms of reflectance [38].

\subsection{TIR Data Processing}

Thermal infrared (TIR) data are used to retrieve Land Surface Temperature (LST) for many ecological and environmental studies on regional and global scales. Although ASTER TIR bands have low spatial resolution $(90 \mathrm{~m})$ compared with shorter wavelength bands $(15-30 \mathrm{~m})$, they add spectral information in addition to the VNIR and SWIR bands. These data are still affected by the issue of mixed pixels of multiple anisothermal objects, altering the results in finer scale studies such as the analysis of urban heat island [39] and stream temperatures [40].

In our case of ASTER Thermal bands (resampled at $15 \mathrm{~m}$ ) were utilized to better discriminate classes with similar spectral response (i.e., built-up area and crop), hardly ever recognizable by means of the most consolidated methods.

To this purpose, the LST was retrieved using the emissivity normalization method (NEM) implemented in the ENVI image processing [37]. The first step in this routine consists in deriving the brightness temperature of each pixel from the pixel radiance. Secondly, the apparent emissivity image is obtained by normalizing the radiance of each pixel to the Planck's curve that is generated from the pixel with the maximum brightness temperature at an emissivity value of 0.96 (i.e., a reasonable hypothesis for soils). Figure 2 shows the positive correlation between NEM processed data and the on-demand Level-2 standard product AST-08 [1] of (a) 24 June 2003 and (b) 14 July 2007. The ASTER Surface Kinetic Temperature product was derived by applying Planck's Law using the emissivity values from the Temperature-Emissivity Separation (TES) algorithm, which uses atmospherically corrected ASTER Surface Radiance (TIR) data. 
Figure 2. NEM processed ASTER data (left), the on-demand Level-2 standard product AST-08 (centre) of (a) 24 June 2003 and (b) 14 July 2007 and the scatter plots showing their correlation (right).

(a)
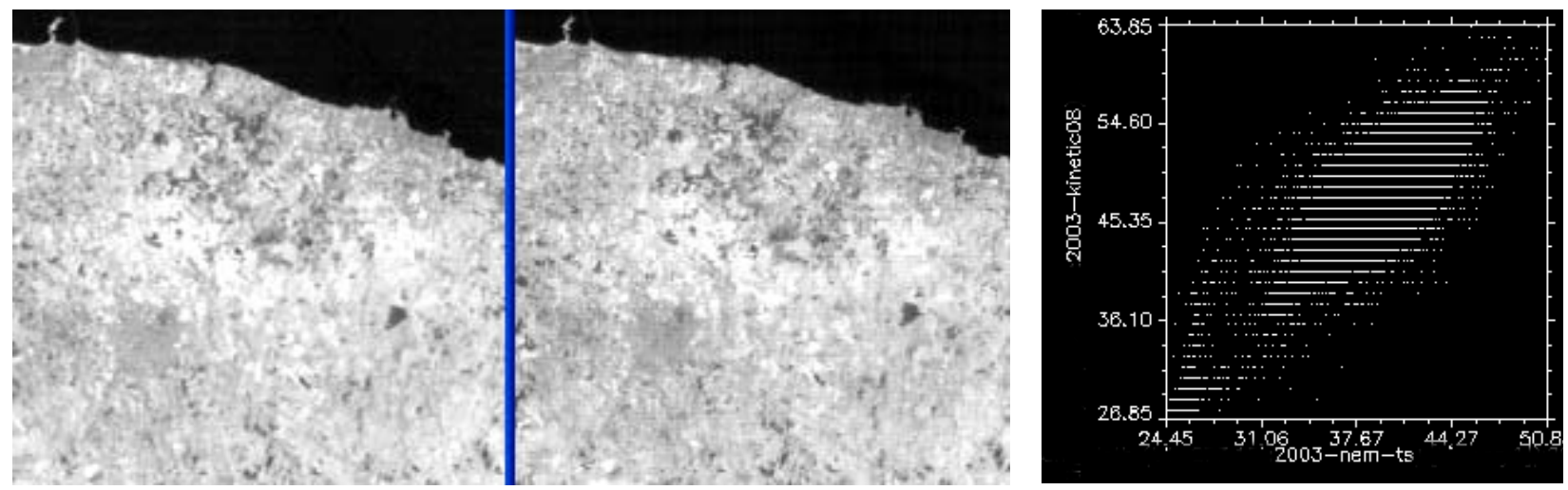

(b)
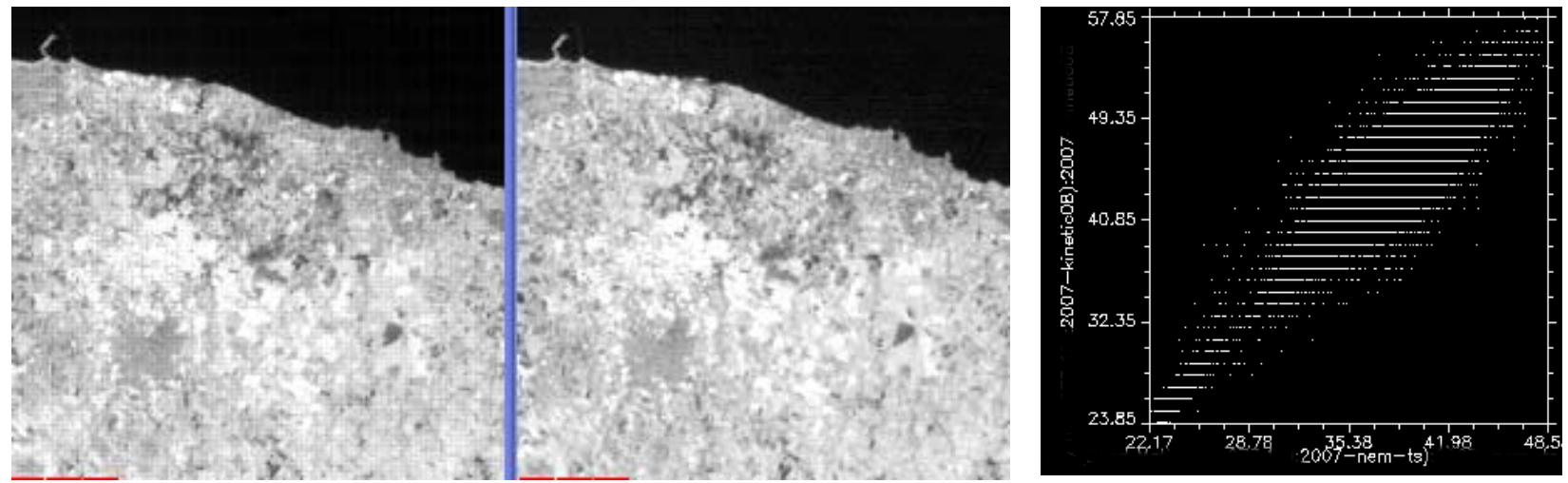

For both 2003 and $2007 \mathrm{~T}_{\mathrm{S}}$ data ENVI Mosaicking Color balancing was then applied in order to match the statistics from the first image to the second one and balance the data range. Gains and offsets were calculated from the fixed image and applied to the adjusted images to ensure the same statistical range for the adjusted images.

The above pre-processing phase was to prepare an additional thermal layer to be used in land cover classification and to supply additional variability for the purpose of enhancing accurate classification of subtle differences between ground objects. Even if temperature data are entirely seasonal, in this work they were used in combination with the "Impervious surface" subarea previously extracted by means of the decision thresholds procedure on VNIR\&SWIR data.

The spatial distribution of Land Surface Temperature $\left(T_{S}\right)$ obtained in this study area mirrors some significant differences in land cover conditions at the time of the year studied (June, 2003-July, 2007). Natural surfaces with large areas covered in olive groves and vineyards, tarpaulins, bare soil and wheat stubble showed higher values of temperature $\left({ }^{\circ} \mathrm{C}\right)$ than artificial ones such as built-up areas, small areas of calc-tufa and marble and water bodies (Figure 3). The reasons for this phenomenon are rather complex if considering the specific microclimatic patterns related to urban and rural morphology [41]. The physical effect emerged in this work was mainly due to the relationship between thermal inertia and surface temperature of various material types at determined day and night hours. Such assumption was studied by Chen et al. [42] who obtained negative correlation coefficients between urban heat island intensity and thermal inertia, explaining that while the surface temperature of a material with a 
small thermal inertia such as bare soil or water can be raised rapidly, the surface temperature of a material with a higher thermal inertia such as a building can be raised slowly. Moreover, the correlation degree varies depending on time, season and surface type.

Further studies are foreseen within and around the present test area to better validate these hypothesis by comparing in situ surface measures and their corresponding air temperatures based on on-demand Level 2 ASTER TIR data.

Figure 3. Spatial distribution of Land Surface Temperature (TS) in the subsets ASTER 2003 and 2007 data.

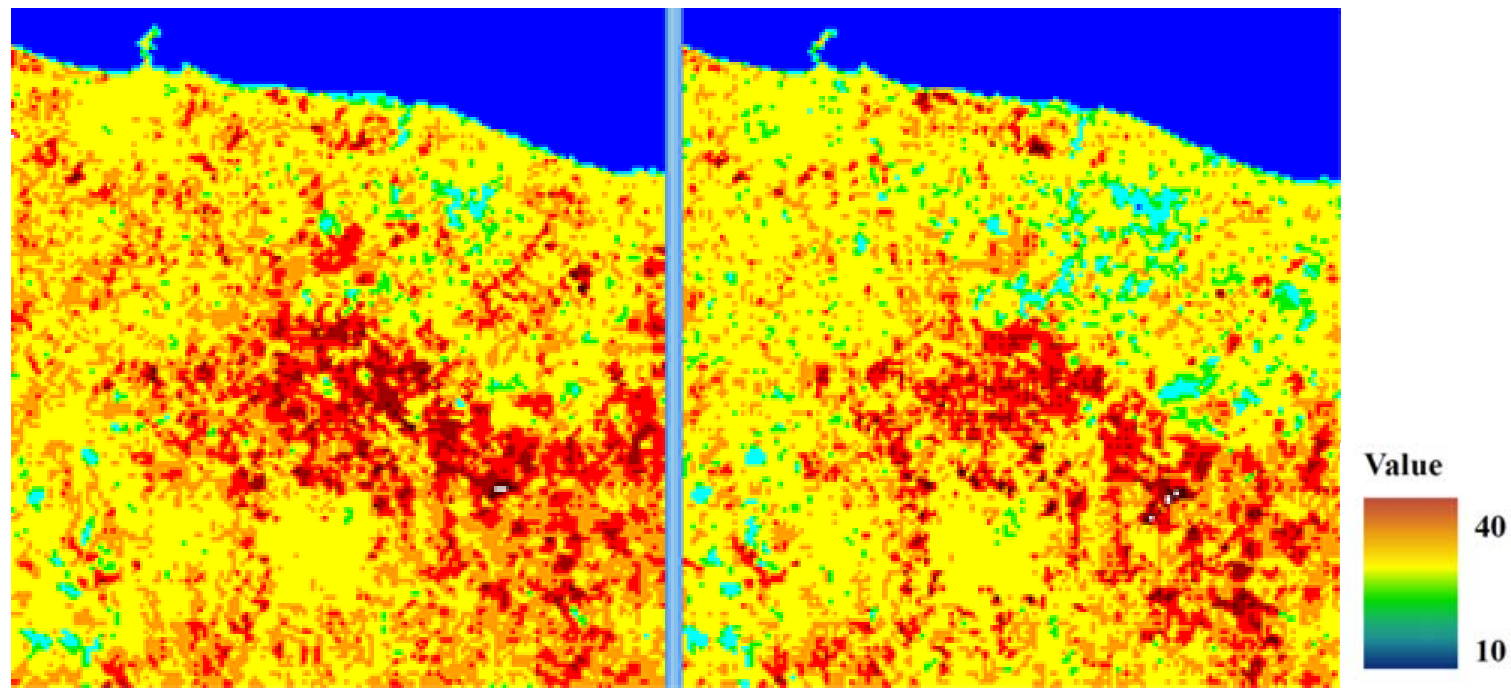

\section{Class-Oriented Classification Method}

This stage included various tasks such as land cover nomenclature and training sample definition as well as the assessment of features identification procedures, both spectral and object-based, to overcome overlapping among classes with similar spectral response.

After choosing a number of classes that best represented the analyzed territory, the Iterative Self Organizing Data Analysis (ISODATA) on the VNIR-SWIR bands was adopted as comparison method among the most consolidated approaches of image classification This method uses spectral distance and iteratively classifies pixels, redefines the criteria for each class, classifying again, so that the spectral distance patterns in the data gradually emerge [43].

In this step homogeneous spatial elements on satellite images were automatically identified. Seven spectral clusters were generated with the following parameters: convergence threshold (95\%), maximum number of merge pairs (2), minimum class standard deviation (1), minimum number of pixels in a class (100), and maximum number of iterations (10). Clusters containing mixed classes were separately reclassified in order to further extract individual classes and obtain an overall classification accuracy of 73\% (2003) and 71\% (2007). Seven classes were next defined for labelling (as shown in Table 1): Water, Urban, Rock, Bare soil, Agriculture, Forest and Spontaneous vegetation. As a first examination of the results, it can be inferred that, besides a general low level of global accuracy, in both data there were several misclassifications and overlapping errors among classes with similar spectral response (i.e., "Urban", "Rock" and "Bare soil”). 
To reduce misclassifications for the above land cover classes and to produce a more accurate spectral separability, a class oriented strategy with a class-oriented approach was then implemented for land cover classification. Figure 4 above shows the workflow of the method.

Table 1. Land cover classification proposal.

\begin{tabular}{ll}
\hline \multicolumn{1}{c}{ Land cover class } & \multicolumn{1}{c}{ Description } \\
\hline Water & $\begin{array}{l}\text { Permanent open water, lakes, reservoirs } \\
\text { Residential, commercial services, industrial, transportation, } \\
\text { Crban }\end{array}$ \\
communications, mixed urban or built-up land \\
Rock & Outcrop and extraction (quarries, sand and gravel pits) \\
Bare soil & Convegetated fields, sand \\
Agriculture & Deciduous forest land, evergreen forest land, mixed forest land, \\
Forest & orchards, groves, vineyards \\
Spontaneous vegetation & sparsely vegetated areas \\
\hline
\end{tabular}

The operational strategy of this work was organized as a combination of pixel-based and object-based procedures to obtain the main distinction between vegetated and non vegetated areas. The pixel-based procedure was related to Vegetation Index (VI) and Surface Temperature $\left(\mathrm{T}_{\mathrm{S}}\right)$ retrieval algorithms, while ENVI Feature Extraction was used as object-based approach [37], because it is more flexible in the types of features to be extracted from single or multichannel imagery. It allows an iterative approach of segmentation which is supported by the previsualization of the output. Users define a single parameter (Scale) and can later refine the segments obtained, by applying in this study the K Nearest Neighbor classification method (K parameter $=3$ ). Hence, in the first step, geometrically and radiometrically corrected VNIR-SWIR stacks and $\mathrm{T}_{\mathrm{S}}$ were processed separately.

The Soil Adjusted Vegetation Index (SAVI), designed to minimize the effects of soil background [44], was computed as a means to gather information on the vegetation cover. SAVI was applied to the ASTER data set using the following equation:

$$
S A V I=\left[\frac{(N I R-R E D)}{(N I R+R E D+L)}\right] \times(1+L)
$$

where $\mathrm{L}$ is an adjustment factor.

Similarly to the Normalized Difference Vegetation Index (NDVI), the near infrared and red bands were used in the calculation of SAVI, but with the addition of an adjustment factor (L), which varies between zero and one. In this case an adjustment factor of 0.5 was used, which resulted in reducing soil influences considerably; for, it is the most widely used adjustment factor for intermediate vegetation cover analysis. Two SAVI qualitative subclasses were created, namely SAVI 1 and SAVI 2 for low and high canopy vitality.

First, the pixels with low values of SAVI 1 (Threshold $<0.1$ ) were masked out from the entire image stack by deriving the "Non vegetation" macroclass. "Water" and "Bare soil" land cover objects were first extracted by means of edge-based segmentation (Scale $=60$ ). Edges are regarded as boundaries among image objects and they are located where changes in values occur. The unclassified 
areas (named "Impervious areas") were left to be examined afterwards by analyzing them jointly with the $\mathrm{T}_{\mathrm{S}}$ image output.

Figure 4. Workflow of the procedures in the class-oriented approach to features extraction.

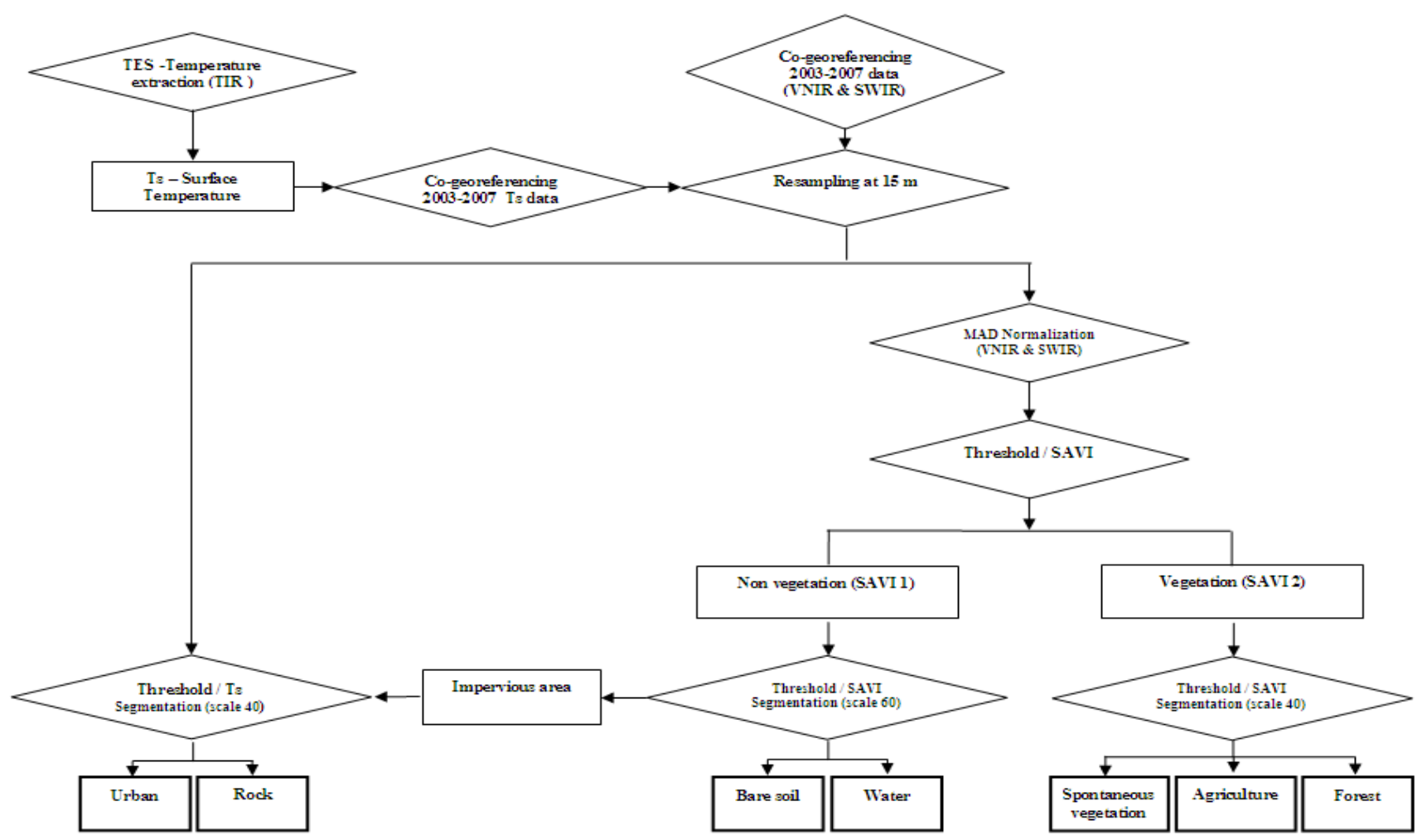

Then, the SAVI 2 image area was segmented (Scale = 40) in "Spontaneous vegetation", "Agriculture" and "Forest" respectively by combining regions of interest containing spatial, spectral (brightness and colour), and/or texture characteristics with the derived information of canopy vitality (medium: $0.1<$ Threshold $>0.3$; high: $0.3<$ Threshold $>0.6$; very high: Threshold $>0.6$ ).

The last step regarded "Urban" and "Rock" land cover extraction. Many studies proved the low performance of the most consolidated pixel-based classification methods [45]. The Normalized Difference Built-up Index (NDBI) method, which only makes use of spectral patterns, was found to be unable to map peripheral urban areas where barren or fallow land is widespread, which is a common situation in arid regions. The OBIA approach works particularly well on VHR image data, while on medium spatial resolution ASTER data some inconsistencies are still remain if only VNIR\&SWIR channels information are analysed. In this territorial contexts groups of objects with equivalent spectral and texture characteristics (as "Urban/Built-up areas" with "Rock/Quarries") may be confused with barren lands. For the purposes of our study, the contribution of $\mathrm{T}_{\mathrm{S}}$ data was considered, showing that the spatial distribution and boundaries among image objects were apparent and able to differentiate objects with similar behaviour.

A supplementary dataset of unclassified pixels, named "Impervious areas" and produced during the "Non vegetation" work, was masked out from geo-referenced $\mathrm{T}_{\mathrm{S}}$ data. Finally, the segmentation of "Urban" and "Rock" (Scale = 40) objects was easily executed by considering the threshold values determined by their different thermal behaviour. To such purpose, every value range was obtained by 
means of the Density Slicing technique, commonly used to enhance the information gathered from an individual brightness band.

\section{Results}

The class-oriented LC (Land Cover) classification of ASTER 2003 and ASTER 2007 data is shown in Figure 5. Classification results (Tables 2-3) were evaluated with overall accuracy indexes derived by testing samples with 30 observations per class (ground truth) in order to derive unbiased accuracy measures. Data airborne orthoimages acquired between 2000 and 2004 were used as reference to attest the accuracy of the LC 2003 map, while the interactive interpretation of Google Earth tools was preferred to validate LC 2007 results. The labelling protocol consisted in assigning the "ground truth" to each testing observation through visual interpretation of reference data.

Figure 5. LC classes after Class-oriented classification on the ASTER data subsets of 24 June, 2003 (left) and 14 July, 2007 (right).
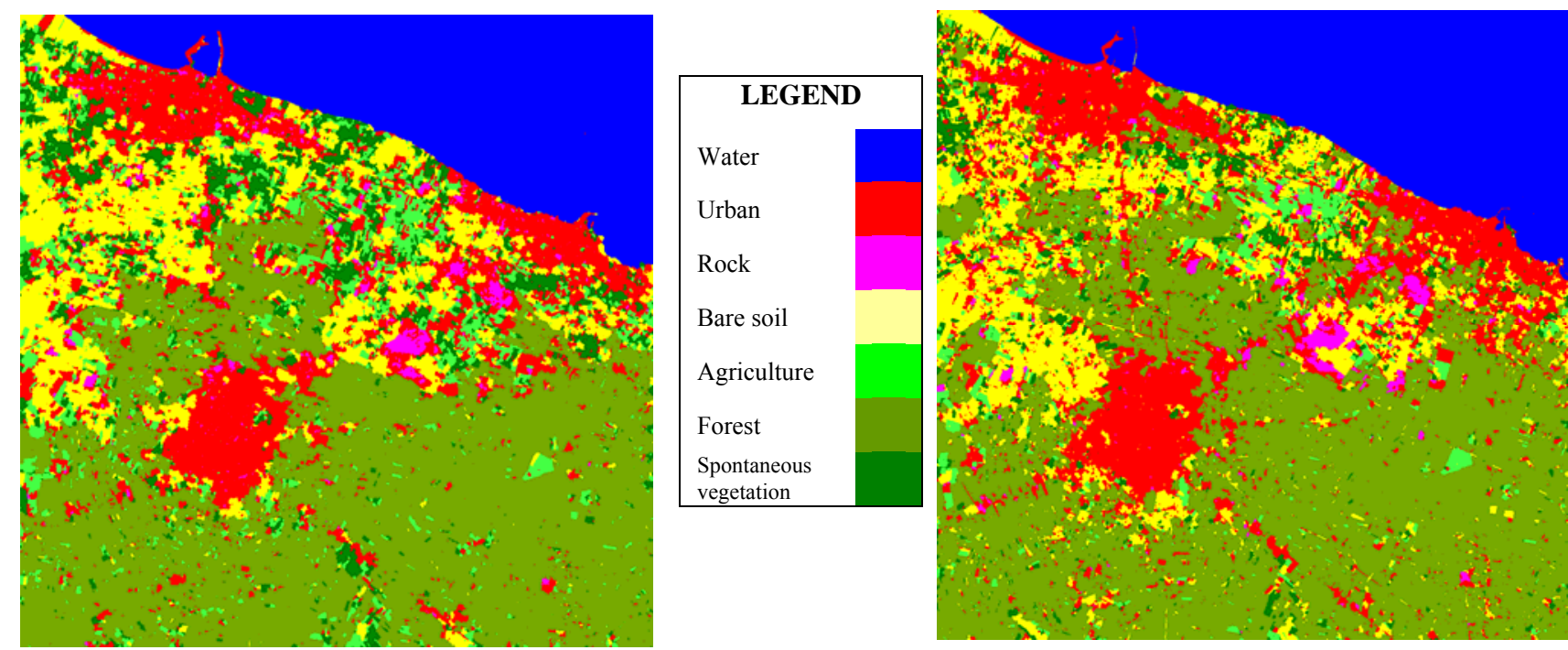

The hybrid classification results showed the possibility to distinguish objects and to generate semantic classes, with overall classification accuracy values of 92.48\% (2003) and 92.84\% (2007).

There were various causes for obvious "classification" errors, with significant difficulty in distinguishing between different artificial areas. For instance, "Rock" objects showed some characteristics similar to those of "Urban" and "Bare soil" classes, which may be imputed to the textured pattern and their orientation towards the sunlight. On the other hand, different base data (VNIR-SWIR and TIR) were used for any of these three classes. While the "Bare soil" class objects were derived from a SAVI index subclass, "Urban" and "Bare soil" class objects with different latent heat were obtained by means of a segmentation approach and, as a consequence, were dependent mainly on the contribution of the spatial and textural distribution of Ts across the study area. For spectral separable classes, such as the vegetation features of "Spontaneous vegetation", "Agriculture" and "Forest", a good recognition rate was obtained. Semantic classes that present errors often contain different class-types causing a partly incorrect representation. For instance, an "Agriculture" class can 
include trees or grass. Segmentation distinguishes between these objects and classifies trees or grass depending on the object-level.

Table 2. Results (Percentages) from the accuracy assessment process (ASTER 2003).

\begin{tabular}{|c|c|c|c|c|c|c|c|c|c|c|c|}
\hline \multirow[b]{2}{*}{ LC Classes } & \multicolumn{11}{|c|}{ Ground Truth } \\
\hline & Urban & $\begin{array}{l}\text { Spont. } \\
\text { vegetation }\end{array}$ & Water & $\begin{array}{l}\text { Bare } \\
\text { soil }\end{array}$ & Agricult. & Rock & Forest & $\begin{array}{l}\text { Prod. } \\
\text { Acc. }\end{array}$ & $\begin{array}{l}\text { User } \\
\text { Acc. }\end{array}$ & Commission & Omission \\
\hline Urban & 89.72 & 7.78 & 0.00 & 5.75 & 0.05 & 23.04 & 1.06 & 89.72 & 78.48 & 21.52 & 10.28 \\
\hline $\begin{array}{l}\text { Spont. } \\
\text { vegetation }\end{array}$ & 0.45 & 74.91 & 0.00 & 1.51 & 3.44 & 0.00 & 0.51 & 74.91 & 93.86 & 6.14 & 25.09 \\
\hline Water & 0.00 & 0.00 & 100.00 & 0.00 & 0.00 & 0.00 & 0.00 & 100.00 & 100.00 & 0.00 & 0.00 \\
\hline Bare soil & 0.82 & 7.86 & 0.00 & 91.04 & 18.97 & 1.26 & 0.09 & 91.04 & 84.73 & 15.27 & 8.96 \\
\hline Agriculture & 0.53 & 7.83 & 0.00 & 1.70 & 76.34 & 0.44 & 1.18 & 76.34 & 57.50 & 42.50 & 23.66 \\
\hline Rock & 8.48 & 2.79 & 0.00 & 2.6 & 0.00 & 75.26 & 0.00 & 75.26 & 79.84 & 20.16 & 27.74 \\
\hline Forest & 0.00 & 1.62 & 0.00 & 0.00 & 1.20 & 0.00 & 97.17 & 97.17 & 99.51 & 0.49 & 2.83 \\
\hline
\end{tabular}

Table 3. Results (Percentages) from the accuracy assessment process (ASTER 2007).

\begin{tabular}{|c|c|c|c|c|c|c|c|c|c|c|c|}
\hline \multirow[b]{2}{*}{ LC Classes } & \multicolumn{11}{|c|}{ Ground Truth } \\
\hline & Urban & $\begin{array}{l}\text { Spont. } \\
\text { vegetation }\end{array}$ & Water & $\begin{array}{l}\text { Bare } \\
\text { soil }\end{array}$ & Agricult. & Rock & Forest & $\begin{array}{l}\text { Prod. } \\
\text { Acc. }\end{array}$ & $\begin{array}{l}\text { User } \\
\text { Acc. }\end{array}$ & Commission & Omission \\
\hline Urban & 98.51 & 4.00 & 0.00 & 5.56 & 2.35 & 19.73 & 2.56 & 98.51 & 71.63 & 28.37 & 1.49 \\
\hline $\begin{array}{l}\text { Spont. } \\
\text { vegetation }\end{array}$ & 0.00 & 69.69 & 0.00 & 4.56 & 0.60 & 0.00 & 0.47 & 69.69 & 82.15 & 17.85 & 30.31 \\
\hline Water & 0.00 & 0.00 & 100.00 & 0.00 & 0.00 & 0.00 & 0.00 & 100.00 & 100.00 & 0.00 & 0.00 \\
\hline Bare soil & 0.13 & 5.39 & 0.00 & 82.72 & 1.61 & 0.09 & 1.95 & 82.72 & 84.08 & 15.92 & 17.28 \\
\hline Agriculture & 0.00 & 0.00 & 0.00 & 0.40 & 95.27 & 0.24 & 1.10 & 95.27 & 86.74 & 13.26 & 4.73 \\
\hline Rock & 1.36 & 0.00 & 0.00 & 0.00 & 0.17 & 79.84 & 0.00 & 79.84 & 97.88 & 2.12 & 20.16 \\
\hline Forest & 0.00 & 20.93 & 0.00 & 6.75 & 0.00 & 0.09 & 93.93 & 93.93 & 97.03 & 2.97 & 6.07 \\
\hline
\end{tabular}

Image pairs of consecutive dates were compared by overlaying the thematic LC maps and cross-tabulating the LC statistics, by using the Change Detection Statistics routine to compile a detailed tabulation of changes between two Classification images. The changes identified in this way significantly differ from a simple differentiation between the two images.

While the statistics report includes a class-by-class Image Difference, the analysis primarily focuses on the initial state classification changes, i.e., for each Initial State class the analysis identifies the classes into which pixels changed in the final state image. Changes can be reported as pixel counts, percentages, and areas. In addition, a special type of mask image (classification masks) that provides a spatial context for the tabular report can be produced [37]. The Class Changes row indicates the total number of initial state pixels that changed classes. The Image Difference row is the difference in the total number of equivalently classed pixels in the two images, computed by subtracting the Initial State Class Totals from the Final State Class Totals. A positive Image Difference indicates the class size has increased. According to the statistics in Table 4, during the period from 2003 to 2007, "Urban", 
"Rock" and "Forest" classes showed a comparatively small positive increase. This is due to urbanization phenomena as much as to the expansion of mining and orchard plantations within a relatively short period of investigation. The increase in barren lands also results from wheat harvesting. A bigger decrease was noted in sparsely vegetated and agricultural areas caused by their conversion to the classes with positive balance (Figure 6A-6B).

Figure 6. (a) Image difference resulting from post-classification comparison of two independently classified images (Black-Change, White-No Change); (b) Subset area of land cover transformations identified on ASTER 2003 and 2007 data.

(a)

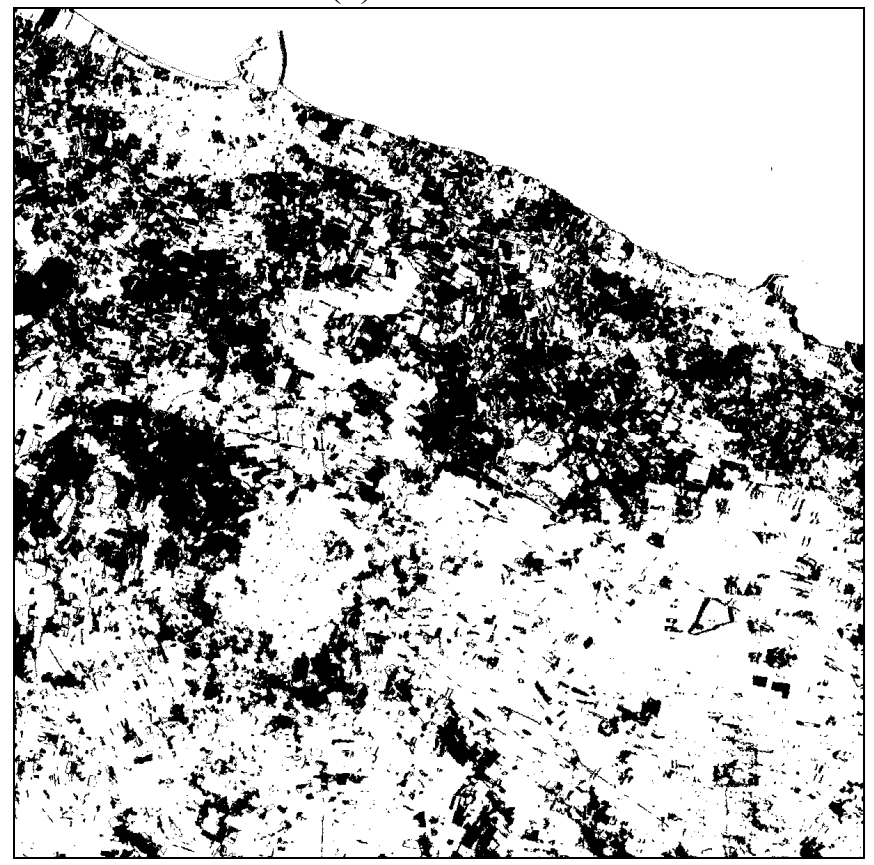

(b)

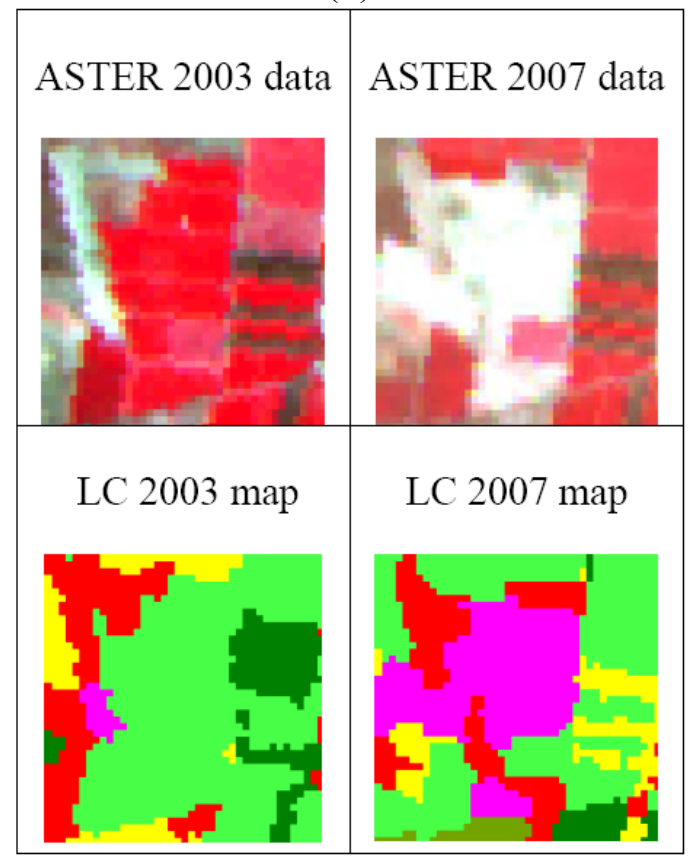

Finally, to validate the exactness of the LC change procedure the Two-Step Approach to Change Detection Accuracy Assessment [46] was performed deriving ground truth samples with the interactive interpretation of Google Earth tools.

The first step in this process is just to assess the accuracy of the areas that changed between the two time periods in question, by conducting a single-date accuracy assessment only on the areas that changed between time 1 and time 2. The sampling procedure is similar to that of a traditional single-date accuracy assessment with the requisite number of samples per land cover class selected using a chosen sampling strategy from the map area. The computation of the Error Matrix of the Accuracy on the 2007 Change Areas (Table 5) was carried out deriving ground truth samples with the interactive interpretation of Google Earth tools. The second step in this process is aimed at change/no change validation by determining a change/no change $(2 \times 2)$ matrix (Table 6). In this case the sampling is only performed to assess the change/no change. Treating the map as a binary or two-class scheme (change/no change), a simpler sampling technique than the multinomial situation of a complete change detection error matrix is required [46]. 
Table 4. Matrices of land cover (LC) and changes (Percentages) from 2003 to 2007.

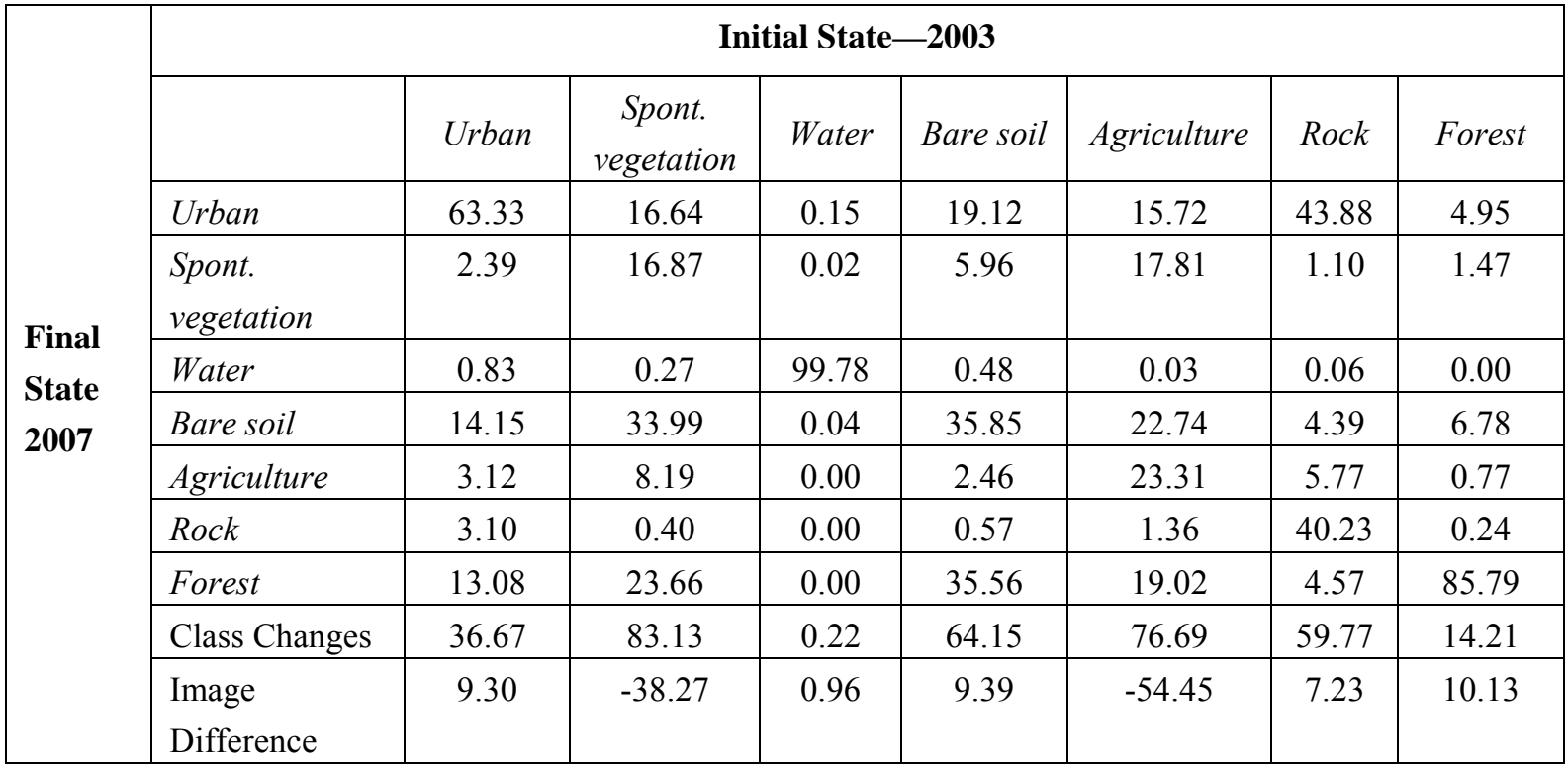

Table 5. Error matrix of the accuracy on the 2007 change areas.

\begin{tabular}{|r|c|c|c|c|c|c|c|c|c|}
\hline \multirow{2}{*}{\begin{tabular}{|} 
MAP \\
\cline { 2 - 11 }
\end{tabular}} & Urban & $\begin{array}{c}\text { Spont. } \\
\text { vegetation }\end{array}$ & Water & $\begin{array}{c}\text { Bare } \\
\text { soil }\end{array}$ & Agr. & Rock & Forest & $\begin{array}{c}\text { Prod. } \\
\text { Acc. } \%\end{array}$ & $\begin{array}{c}\text { User } \\
\text { Acc. } \%\end{array}$ \\
\hline Urban & 1000 & 125 & 0 & 96 & 64 & 181 & 106 & 93.37 & 63.61 \\
\hline $\begin{array}{r}\text { Spont. } \\
\text { vegetation }\end{array}$ & 0 & 1644 & 0 & 33 & 57 & 0 & 0 & 75.48 & 94.81 \\
\hline Water & 0 & 0 & 0 & 0 & 0 & 0 & 0 & 100.00 & 100.00 \\
\hline Bare soil & 3 & 283 & 0 & 3096 & 64 & 7 & 1514 & 95.50 & 62.33 \\
\hline Agriculture & 0 & 123 & 0 & 6 & 1119 & 6 & 0 & 85.35 & 89.23 \\
\hline Rock & 52 & 0 & 0 & 0 & 2 & 602 & 4 & 73.87 & 91.21 \\
\hline Forest & 16 & 3 & 0 & 11 & 5 & 19 & 17 & 1.04 & 23.94 \\
\hline Class Total & 1071 & 2178 & 0 & 3242 & 1311 & 815 & 1641 & & \\
\hline
\end{tabular}

Table 6. Final change/no change matrix.

\begin{tabular}{|l|c|c|c|c|}
\hline \multirow{2}{*}{ MAP } & \multicolumn{4}{|c|}{ REFERENCE } \\
\cline { 2 - 5 } & Change & No Change & Prod. Acc. Total & Prod. Acc. \% \\
\hline Change & 7754 & 1095 & 8849 & 69.64 \\
\hline No Change & 3381 & 11699 & 15080 & 91.44 \\
\hline User Acc. Total & 11135 & 12794 & 23929 & \\
\hline User Acc. \% & 87.63 & 77.58 & & \\
\hline \multicolumn{5}{|c|}{ Overall Accuracy $=81.29 \% ;$ Kappa Coefficient $=0.62$} \\
\hline
\end{tabular}

\section{Conclusions}

In this work an efficient class-oriented strategy was developed on ASTER data to detect changes in a coastal Italian territory with a predominantly agricultural vocation. In the first stage of this work a full pixel-based method (i.e., ISODATA) was implemented with a resulting overall accuracy 
of 73\% (2003) and 71\% (2007). "Urban", "Rock" and "Bare soil" were proved to be the most problematic classes due to the presence of misclassifications and overlapping errors.

In literature object-based classification has been demonstrated to improve results of high detailed land cover classes. Yet, with the sole segmentation it was impossible to ensure that every object derived from the homogeneity criteria contained only one class of pixels. This mixed-object effect worsens along the edge or borders of two cover types or with similar spectral response. Therefore, a class-oriented approach, with the combined use of both the pixel-based and object-based methods, increased the overall classification result accuracy up to $92.48 \%$ (2003) and 92.84\% (2007), enabling the following accurate and coherent LC changes analysis. Hence, natural covers were investigated on VNIR-SWIR bands and only two difficult-to-separate covers ("Urban" and "Rock") required to be treated by managing TIR channel data. For the purposes of our study, the contribution of the $\mathrm{T}_{\mathrm{S}}$ data was also analyzed, showing that the spatial distribution and the boundaries among image objects were more apparent. Evident improvements were found above all for "Urban" class objects. Some discrepancies still persisted in "Bare soil " regions, confused with "Spontaneous vegetation" and "Agriculture" ones. This was due to the specific microclimatic patterns related to urban and rural morphology. Further works are foreseen to investigate this aspect by analyzing TIR channel data in LC classifications for different seasonal and annual acquisitions, in order to widen the analysis on more LC thematic classes. The LC changes results demonstrated the realistic trends of anthropic intervention across the entire study area. This also resulted from the accurate radiometric corrections performed on raw data.

\section{Acknowledgments}

The authors would like to thank the two anonymous reviewers for their comments, which helped to improve the quality of this paper.

\section{References}

1. Abrams, M.; Hook, S.; Ramachandran, B. ASTER Users Handbook, 2nd edition; NASA JPL: Jet Propulsion Laboratory/California Institute of Technology: Pasadena, CA, USA, 2002. Available online: http://asterweb.jpl.nasa.gov/content/03_data/04_Documents/aster_user_guide_v2.pdf (accessed on 11 June 2009).

2. Yamaguchi, Y.; Kahle, A.B.; Tsu, H.; Kawakami, T.; Pniel, M. Overview of advanced spaceborne thermal emission and reflection radiometer (ASTER). IEEE T. Geosci. Remote Sens. 1998, 36, $1062-1071$.

3. Chen, Y.; Shi, P.; Fung, T.; Wang, J.; Li, X. Object-oriented classification for urban land cover mapping with aster imagery. Int. J. Remote Sens. 2007, 28, 4645-4651.

4. Viet, A.H.; Williams, M.; Manning, D. Remote-Sensing Monitoring of Desertification Using ASTER and ENVISAT ASAR: Case Study at Semi-Arid Area of Vietnam. International Symposium on Geoinformatics for Spatial Infrastructure Development in Earth and Allied Sciences, Ho Chi Minh City, Vietnam, November 9-11, 2006. 
5. Zhou, W.; Troy, A.; Grove, M. Object-based land cover classification and change analysis in the baltimore metropolitan area using multitemporal high resolution remote sensing data. Sensors 2008, 8, 1613-1636.

6. Wang, L.; Sousa, W.P.; Gong, P. Integration of object-based and pixel-based classification for mapping mangroves with IKONOS imagery. Int. J. Remote Sens. 2004, 25, 5655-5668.

7. Wilson, J.S.; Clay, M.; Martin, E.; Stuckey, D.; Vedder-Risch, K. Evaluating environmental influences of zoning in urban ecosystems with remote sensing. Remote Sens. Environ. 2003, 86, 303-321.

8. Realmuto, V. Separating the Effects of Temperature and Emissivity: Emissivity Spectrum Normalization. Proceedings of Second Annual Airborne Earth Science Workshop; Abbott, E.A., Ed.; JPL - Jet Propulsion Laboratory Publication: Pasadena, CA, USA, 1990; pp. 31-35.

9. Townshend, J.R.G. Land cover. Int. J. Remote Sens. 1992, 13, 1319-1328.

10. Donnay, J.P. Use of remote sensing information in planning. In Geographical Information and Planning; Stillwell, J., Geertman, S., Openshaw, S., Eds.; Springer-Verlag: Berlin, Germany, 1999; pp. 242-260.

11. Aplin, P.; Atkinson, P.; Curran, P. Per-field classification of land use using the forthcoming very fine resolution satellite sensors: problems and potential solutions. In Advances in remote sensing and GIS analysis; Atkinson, P., Tate, N., Eds.; Wiley \& Son: Chichester, UK, 1999; pp. 219-239.

12. Caprioli, M.; Tarantino, E. Accuracy assessment of per-field classification integrating very fine spatial resolution satellite sensors imagery with topographic data. J. Geospat. Eng. 2001, 3, 127-134.

13. Blaschke, T.; Lang, S.; Lorup, E.; Strobl, J.; Zeil, P. Object-oriented image processing in an integrated GIS/remote sensing environment and perspectives for environmental applications. In Environmental Information for Planning, Politics, and Public, Vol. II; Cremers, A., Greve, K., Eds.; Metropolis Verlag: Marburg, Germany, 2000; pp. 555-570.

14. Baatz, M.; Schaepe, A. Multiresolution segmentation — an optimization approach for high quality multiscale image segmentation. In Angewandte Geographische Informationsverarbeitung; Strobl, J., Blaschke, T., Eds.; Wichmann: Heidelberg, Germany, 2000; Volume 2, pp. 12-23.

15. Caprioli, M.; Tarantino, E. Identification of land cover alterations in the Alta Murgia National Park (Italy) with VHR satellite imagery. Int. J. Sustain. Dev. Plann. 2006, 1, 1743-7601.

16. Gorte, B. Probabilistic Segmentation of Remotely Sensed Images. ITC Publication Series 63; ITC: Enschede, The Netherlands, 1998.

17. Gao, J. Mapping of land degradation from ASTER data: a comparison of object-based and pixel-based methods. GISci. Remote Sens. 2008, 45, 1548-1603.

18. Dehvari, A.; Heck, R.J. Comparison of object-based and pixel based infrared airborne image classification methods using DEM thematic layer. J. Geogr. Reg. Plann. 2009, 2, 86-96.

19. Antunes, A.F.B.; Lingnau, C.; Da Silva, J.C. Object oriented analysis and Semantic Network for High Resolution Image Classification. In Proceedings of the Anais XI SBSR; Belo Horizonte, Brazil, April 5-10, 2003; pp. 273-279.

20. Hay, G.J.; Castilla, G. Geographic object-based image analysis (GEOBIA): Paradigm shift or new methods? In Object-Based Image Analysis: Spatial Concepts for Knowledge-Driven Remote 
Sensing Applications; Blaschke, T., Lang S., Hay, G., Eds.; Springer-Verlag: Berlin, Germany, 2008, pp. 75-89.

21. Lizarazo, I.; Elsner, P. From pixels to grixels: a unified functional model for geographic object—based image analysis. Proceedings of the GEOBIA 2008-Pixels, Objects, Intelligence. GEOgraphic Object Based Image Analysis for the 21st Century; Hay, G.J., Blaschke, T., Marceau, D., Eds.; University of Calgary: Calgary AB, Canada, August 5-08, 2008; ISPRS: Calgary, AB, Canada, 2008; Vol. No. XXXVIII-4/C1, 1682-1777.

22. Singh, A. Digital change detection techniques using remotely-sensed data. Int. J. Remote Sens. 1989, 6, 989-1003.

23. Macleod, R.D.; Congalton, R.G. A Quantitative Comparison of Change Detection Algorithms for Monitoring Eelgrass from Remotely Sensed Data. Photogramm. Eng. Remote Sens. 1998, 64, 207-216.

24. Coppin, P.; Bauer, M. Digital change detection in forest ecosystems with remote sensing imagery. Remote Sens. Rev. 1996, 13, 207-234.

25. Deer, P.J. Digital Change Detection Techniques: Civilian and Military Applications. Technical report. Information Technology Division. DSTO Electronics and Surveillance Research Laboratory: Salisbury, SA, Australia, 1995. Available online: http://hdl.handle.net/1947/4389 (accessed on 2 July 2009).

26. Scheidt, S.; Ramsey, M.; Lancaster, N. Radiometric normalization and image mosaic generation of ASTER thermal infrared data: An application to extensive sand sheets and dune fields. Remote Sens. Environ. 2008, 112, 920-933.

27. Yang, X.; Lo, C. Relative radiometric normalization performance for change detection form multidate satellite images. Photogramm. Eng. Remote Sens. 2000, 66, 967-980.

28. Furby, S.L.; Campbell, N.A. Calibrating images from different dates to 'like-value' digital counts. Remote Sens. Environ. 2001, 77, 186-196.

29. Jensen, J.R. Introductory Digital Image Processing: a Remote Sensing Perspective, 2nd edition; NJ Prentice-Hall: New York, NY, USA, 1996.

30. Schott, J.R.; Salvaggio, C.; Volchok, W.J. Radiometric scene normalization using pseudo-invariant features. Remote Sens. Environ. 1998, 26, 1-16.

31. Hall, F.G.; Strebel, D.E.; Nickeson, J.E.; Goetz, S.J. Radiometric rectification: Toward a common radiometric response among multidate, multisensor images. Remote Sens. Environ. 1991, 35, $11-27$.

32. Du, Y.; Teillet, P.M.; Cihlar, J. Radiometric normalization of multitemporal high-resolution images with quality control for land cover change detection. Remote Sens. Environ. 2002, 82, $123-134$.

33. Canty, M.J.; Nielsen, A.A.; Schmidt, M. Automatic radiometric normalization of multitemporal satellite imagery, Remote Sens. Environ. 2004, 91, 441-451.

34. Canty, M.J. CDSAT-ENVI plug-ins for change detection in multispectral satellite imagery. Available online: http://www.fz-juelich.de/ste/remote_sensing (accessed on July 2007).

35. Nielsen, A.A.; Conradsen, K.; Simpson, J.J. Multivariate alteration detection (MAD) and MAF post-processing in multispectral bitemporal image data: new approaches to change detection studies. Remote Sens. Environ. 1998, 64, 1-19. 
36. Nussbaum, S.; Niemeyer, I. Automated extraction of change information from multispectral satellite imagery. Esarda Bulletin 2007, 36, 19-25.

37. ITT Visual Information Solutions. ENVI - Environment for Visualizing Images, Version 4.5, 2008. Available online: http://www.ittvis.com/ (accessed on 11 May 2009).

38. Tarantino, E.; Figorito, B.; Caprioli, M. Comparison of radiometric calibration methods for change detection analysis on LANDSAT ETM+ and ASTER data. Bull. Geodesy Geomatics 2008, 2, 89-106.

39. Lu, D.; Weng, Q. Spectral mixture analysis of ASTER images for examining the relationship between urban thermal features and biophysical descriptors in Indianapolis, Indiana, USA. Remote Sens. Environ. 2006, 104, 157-167.

40. Gustafson, W.T.; Handcock, R.; Gillespie, A.R.; Tonooka, H. An image-sharpening method to recover stream temperatures from ASTER images. In Remote Sensing for Environmental Monitoring, GIS Applications and Geology, Proceedings of SPIE 2003, Barcelona, Spain; 9-11 September 2003, Ehlers, M., Ed.; SPIE-International Society for Optical Engineering: Bellingham, WA, USA, 2003; pp. 72-83.

41. Nichol, J. Remote sensing of urban heat islands by day and night. Photogramm. Eng. Remote Sens. 2005, 71, 613-621.

42. Chen, Y.; Du, M.; Dong, R. Correlation between urban heat island effect and the thermal inertia using ASTER data in Beijing, China. IAPRS\&SIS-The International Archives of the Photogrammetry, Remote Sensing and Spatial Information Sciences; Proceedings of the XXI ISPRS Congress, Beijing, China, 3-11 Jul 2008; Vol. XXXVII, Part B7, 1723-1728.

43. Tso, B.; Mather, P. Classification Methods for Remotely Sensed Data; Taylor and Francis: New York, NY, USA, 2001.

44. Huete, A.R. A soil adjusted vegetation index (SAVI). Remote Sens. Environ. 1988, 25, 295-309.

45. Qian, J.; Zhou, Q.; Hou, Q. Comparison of pixel-based and object-oriented classification methods for extracting built-up areas in arid zone. ISPRS Workshop on Updating Geo-spatial Databases with Imagery \& the 5th ISPRS Workshop on DMGISs, Urumchi, Xingjiang, China, August 28-29, 2007.

46. Congalton, R.G.; Green, K. Assessing the Accuracy of Remotely Sensed Data-Principles and Practices, 2nd ed.; CRC Press, Taylor \& Francis Group: Boca Raton, FL, USA, 2009.

(C) 2009 by the authors; licensee Molecular Diversity Preservation International, Basel, Switzerland. This article is an open-access article distributed under the terms and conditions of the Creative Commons Attribution license (http://creativecommons.org/licenses/by/3.0/). 